\title{
Factores de riesgo cardiovascular en enfermos adultos con evento vascular cerebral
}

\author{
Sandra Rodríguez Duarte
}

\begin{abstract}
RESUMEN
El evento vascular cerebral (EVC) tiene más de una causa, en muchos casos se debe a múltiples factores de riesgo cardiovascular (FRCV) que en su mayoría son modificables. Objetivos: identificar y describir los FRCV asociados al EVC en pacientes cardiópatas; caracterizar a la población con EVC de la Unidad Coronaria del Instituto Nacional de Cardiología Ignacio Chávez. Material y métodos: es un estudio descriptivo, transversal y retrospectivo. Mediante la revisión de la base de datos de la Unidad Coronaria se obtuvieron antecedentes y factores de riesgos modificables y no modificables de los pacientes ingresados con EVC en el periodo de septiembre de 2005 a mayo de 2009. El análisis estadístico de datos se realizó mediante Excell ${ }^{\circledR} 2007$ y se expresaron en medidas de resumen. Resultados: se revisaron 134 registros de enfermos con EVC, presentándose una morbilidad del 0.5\%; con predominio del sexo femenino en un 61\%; hipertensión arterial sistémica prevaleció como factor de riesgo cardiovascular con más frecuencia en un 60\% y fibrilación auricular en un 43\%. Únicamente 13 pacientes recibieron tratamiento trombolítico. Conclusión: los FRCV asociados al EVC en pacientes cardiópatas son: hipertensión arterial, fibrilación auricular y diabetes mellitus, mismos que son padecimientos prevenibles o controlables a través de educación para la salud.
\end{abstract}

Palabras claves: EVC, factores-riesgo, cardiovascular, hipertensión arterial sistémica.

\section{Risk factor cardiovascular in patients adult with cerebral vascular event}

\begin{abstract}
Often the cerebral vascular event (EVC) has more than one cause, in many cases is due to multiple cardiovascular risk factors (CRF) in its most modified. Objective: to identify and describe the EVC associated cardiovascular risk factors in patients with heart disease, and characterize the population for CVD in the Coronary Care Unit of the National Institute of Cardiology. Methodology: it is a descriptive, cross-sectional and retrospective. By reviewing the database of the Coronary Care Unit were obtained background and modifiable risk factors and modifiable CVD patients admitted in the period September 2005 to May 2009. Statistical analysis of data using Excel $₫ 2007$ and were expressed in summary measures. Results: we reviewed 134 records of patients with CVD, presenting a morbidity of $0.5 \%$ were females by $61 \%$, the prevailing systemic arterial hypertension as a cardiovascular risk factor more frequent in $60 \%$ and atrial fibrillation by $43 \%$. Only 13 patients received thrombolytic treatment. Conclusion: EVC associated cardiovascular risk factors in patients with heart disease are high blood pressure, atrial fibrillation and diabetes mellitus, these are preventable or controllable diseases through health education.
\end{abstract}

Key words: EVC, risk factors, cardiovascular, hypertension.

Correspondencia: Sandra Rodríguez Duarte. Clínica de Anticoagulantes del Instituto Nacional de Cardiología Dr. Ignacio Chávez. Juan

Badiano \# I, Col. Sección XVI, 14080 México, D.F. 
$\mathrm{E}$ n la actualidad el evento vascular cerebral EVC de origen cardioembólico representa el $27 \%$ de los casos en México y se cálcula que 32,000 personas sufren EVC anualmente en México, ocupando la cuarta causa de muerte en la población general, con predominio al sexo femenino.

La American Heart Association describe al EVC como un término general que se refiere a una alteración neurológica aguda que sigue a la interrupción del suministro de sangre de una parte específica del cerebro.

Existen dos tipos de EVC; el isquémico y el hemorrágico, responsable del 85\% de los casos y consiste en la oclusión de un vaso sanguíneo que interrumpe el flujo a una región específica del cerebro, interfiriendo en las funciones neurológicas dependientes de la región afectada. La ausencia de flujo sanguíneo puede ser atribuida a uno de dos procesos diferentes como: trombosis, por un estrechamiento u oclusión local de una arteria que obstruye el flujo sanguíneo distal; y embolismo o cierre de una arteria por material originado más próximamente al corazón, sistema venoso o arterias proximales.

En cuanto al EVC hemorrágico existen dos grandes subcategorías: hemorragia subaracnoidea, en esta la hemorragia se produce dentro de los espacios situados alrededor del cerebro, y la hemorragia intracraneal donde el sangrado está directamente dentro del parénquima cerebral.

Los factores de riesgo cardiovascular (FRCV) asociados al EVC incluyen a aquellos de tipo no modificables como: edad, género, historia familiar, raza y otros modificables, susceptibles de intervención terapéutica como: hipertensión arterial sistémica (HTA), coronariopatía, dislipidemia, diabetes mellitus (DM) y tabaquismo, entre otros.

Se ha demostrado que la hipercolesterolemía, resistencia a la insulina, hipertensión arterial, diabetes mellitus, obesidad y tabaquismo producen disfunción endotelial al bloqueo de síntesis de oxído nítrico, al activar el estrés oxidativo producce radicales de superóxido; lo cual no sólo provoca vasoconstricción, sino también favorece la adhesión de macrófagos a la pared endotelial, que predispone a trombosis intra-arterial por disminución de producción de proteína $\mathrm{C}$ reactiva y de la actividad fibrinolítica.

Ante un daño endotelial, la interrelación del subendotelio con plaquetas, mecanismos de coagulación y la participación de otros elementos circulantes, constituyen la base fisiopatológica de la trombosis.

Entre las causas del EVC; de origen cardiovascular se encuentran la fibrilación auricular (Fa) y cardiopatía isquémica que inciden en un $45 \%$ de todos los casos, le sigue el infarto al miocardio con un $15 \%$, aneurisma ventricular, enfermedad cardiaca reumática y válvula protésica en un 10\% respectivamente $^{1,8 .}$
El EVC causa una gran variedad de signos y síntomas que dependen de localizar de la lesión, tamaño del área de hipoperfusión o extensión del daño; manifestándose con paresia o parestesia en cara, brazo o pierna, sobre todo de un lado del cuerpo, confusión o cambio en el estado mental, problemas para hablar o entender el habla, dificultad para caminar, mareo, pérdida del equilibrio o coordinación, cefalea repentina e intensa, rígidez leve de nuca, estupor, coma y por último la muerte.

La cantidad de enfermos que son atendidos con EVC en el servicio de Urgencias de la Unidad Coronaria no representó un número considerable; sin embargo, muchos enfermos que han sido atendidos en otra institución por presentar un EVC de origen cardioembólico son referidos al Instituto ingresando por Consulta Externa; por lo tanto, la cantidad de enfermos con EVC que son atendidos por el profesional de enfermería de la Clínica de Anticoagulantes es mucho mayor que el que se reporta en la Unidad Coronaria. Ante esta problemática se requiere identificar y describir los FRCV relacionados con EVC y caracterizar a la población que se atiende en el Instituto. Cabe mencionar que con base en los resultados se identificarán áreas de oportunidad para la participación de enfermería, como parte del equipo de salud, al tener la capacidad para continuar y colaborar en el manejo terapéutico con un enfoque integral, educativo, diagnóstico, monitoreo, prevención y limitación del daño que produce el EVC a la salud.

\section{MATERIAL Y MÉTODOS}

Se realizó un estudio de tipo descriptivo, transversal y retrospectivo de los factores de riesgo cardiovascular de $(n=5,725)$ enfermeros ingresados a la Unidad Coronaria del Instituto Nacional de Cardiología Ignacio Chávez. Se realizó una búsqueda en la base de datos del servicio donde se incluyeron enfermos adultos con diagnóstico de EVC isquémico o hemorrágico de septiembre de 2005 a mayo de 2009; se excluyeron enfermos que fueron referidos a otros institutos o que fallecieron. Se tomaron datos de los expedientes clínicos para analizar las variables de factores de riesgo modificables y no modificables, que se relacionan con la presencia de EVC y los sociodemográficas con el fin de caracterizar a la población.

El análisis estadístico de los datos se realizó mediante Excel ${ }^{\circledR}$ 2007, expresandose en medidas de resumen con promedios y porcentajes.

\section{RESULTADOS}

La muestra quedó constituida por $\mathrm{n}=134$ enfermos que presentaron EVC, por lo que se tiene una incidencia de $2.34 \%$ donde predominó el sexo femenino en un 61.2\%; el rango de edad fue de 58 a 64 años, lo que correspondió al 68\% de los casos. 
Entre las enfermedades cardiológicas asociados al EVC; que se presentaron con mayor frecuencia se encontró a la HTA en un $60.4 \%$ con una presión arterial sistólica media de $133 \pm$ $39 \mathrm{mmHg}$ y una presión arterial media con un promedio de 96 mmHg; en segundo lugar la Fa en un 43.28\%, seguido de la cardiopatía isquémica en 35\% (cuadro 1).

Cuadro 1. Factores de riesgo para EVC.

\begin{tabular}{lcc}
\hline & \multicolumn{2}{c}{$\%(\mathrm{~N}=134)$} \\
& $\mathrm{SI}$ & $\mathrm{NO}$ \\
\hline Enfermedadescardiovasculares & & \\
Hipertensión arterial sistémica & 60.45 & 39.55 \\
Fibrilación auricular & 43.28 & 56.72 \\
Cardiopatíaisquémica & 35.07 & 64.93 \\
Infarto previo mayor a un mes & 19.4 & 80.6 \\
Insuficiencia cardiaca & 19.4 & 80.6 \\
Enfermedades asociadas & & \\
Diabetes mellitus & 21.64 & 78.36 \\
Obesidad & 20.89 & 79.11 \\
Insuficiencia renal crónica & 11.94 & 88.06 \\
Factores de riesgo modificables & & \\
Tabaquismo & 8.95 & 91.05 \\
Alcoholismo & 9.70 & 90.3 \\
\hline
\end{tabular}

Las principales enfermedades asociadas al EVC son la DM en $21.6 \%$ y obesidad en el $20.89 \%$ de los casos. Por otro lado, en el análisis de los factores de riesgo modificables se encontraron en el siguiente orden: la diabetes mellitus se presentó en un $21.64 \%$, obesidad en $20.89 \%$, alcoholismo en $10 \%$ y tabaquismo previo en $32.09 \%$.

En cuanto al tratamiento únicamente el 10\% fue trombolizado, el $40.29 \%$ fue manejado con anticoagulante oral (acenocumarol) y el 33.58\%, con aspirina. También se encontró que un 27\% de los enfermos con EVC requirieron ventilación mecánica asistida púes el padecimiento afecta funciones cerebrales como la respiración.

La morbilidad por EVC fue de $0.5 \%$ de la población total del Servicio de la Unidad Coronaria; sin embargo, considerando sólo los enfermos con EVC el porcentaje de fallecimientos es el 23\% de la muestra.

\section{DISCUSIÓN}

Los factores de riesgo de origen cardiovascular encontrados en esta investigación; son comparables con otros estudios publicados en el mundo, como el realizado por San Clemente y Cira García, donde se encontró como factor de riesgo a la hipertensión arterial en el 63.4 y 56.4\% respectivamente, mientras que en este estudio es del $60 \%$; por lo que existe evidencia que señala a la HTA; como el factor de riesgo previsible más importante en el EVC, tanto de tipo isquémico como hemorrágico; por lo tanto, el buen control de la presión arterial obtenido en las primeras 24 hs influye en la buena evolución del EVC. La existencia de programas bien diseñados y adaptados a la realidad en la atención primaria a la salud para el abordaje y control de la presión arterial, así como del resto de FRCV, hace que sea más sencillo el control de los mismos, aunque todavía un porcentaje muy bajo de hipertensos consiguen objetivos terapéuticos.

Otro factor de riesgo asociado al EVC relevante es la Fa, que se presenta en el 7.6\% en el estudio de Cira y en el 45\% en San Clemente, datos similares a este estudio, donde la incidencia es del $43 \%$. Cabe mencionar, que cuando se combina este trastorno del ritmo con la HTA, ateroesclerosis y edad aumenta la incidencia de EVC.

La edad que predominó en el estudio de Cira García fue de 40 a 69 años, con 25.7\%, mientras que el rango de edad en este estudio de pacientes con EVC osciló entre 58 a 64 años, que representa el 68\% del total de enfermeros con EVC en hospitalización, por lo que se puede decir que también se asocia con la edad.

Otros FRCV asociados a la presencia de EVC de acuerdo a Cira García, Amariles y Gómez es diabetes mellitus, obesidad, tabaquismo, desapego terapéutico, malos hábitos alimenticios y sedentarismo destacando que son aspectos previsibles, por lo tanto, tiene fundamental importancia para que sean estrictamente vigilados por el profesional de enfermería y modificado a través de educación para la salud y cambios de estilo de vida saludable, ya que cuando el enfermo tiene un conocimiento adecuado de los FRCV, hay una disminución de los mismos, se reduce la morbilidad y el reingreso hospitalario.

Al tratarse de un estudio retrospectivo estamos conscientes de la falta de datos acerca de antecedentes familiares de enfermedad cardiovascular, niveles de colesterol y triglicéridos, entre otros; por tanto no se pueden obtener registros sobre la influencia que estos tienen para desarrollar EVC, pero sí podemos decir que el profesional de enfermería basado en sus competencias, debe aplicar las políticas y estratégias para el control y prevención secundaria de esta enfermedad crónica.

Es importante iniciar la movilización y rehabilitación de manera precoz, ya que mejoran las incapacidades producidas por EVC; asimismo, la evaluación de la necesidad de la rehabilitación en fase aguda y conexión con centros de salud para darle continuidad a la asistencia en la convalecencia del paciente al momento del alta.

Por último, de acuerdo a Amariles y Gómez, es necesario la planificación e implementación de programas de prevención secundaria de las complicaciones derivadas de los FRCV, pues los pacientes desconocen la necesidad de modificar sus hábitos de vida y de la importancia del autocuidado, siendo este una oportunidad de mejora. 


\section{CONCLUSIONES}

Los FRCV; son un conjunto de condiciones clínicas que han sido involucradas como importantes detonantes que interactúan en forma sinérgica, causando una aceleración de la progresión en la formación de trombos. Por lo tanto, tienen un valor predictivo de alto riesgo para enfermedad trombótica y en este caso de EVC.

Los factores de riesgo y enfermedades cardiológicas asociados al EVC que se encontraron en el Servicio de Unidad Coronaria del Instituto Nacional de Cardiología Ignacio Chávez son padecimientos prevenibles como: hipertensión arterial, obesidad, y diabetes mellitus. Por lo que son necesarios programas de consultoría de enfermería enfocados a prevención primaria y secundaria de los FRCV en el EVC, para mejorar la asistencia y la calidad de vida. El profesional de enfermería tiene la posibilidad de establecer comunicación con la persona en casí todo momento para proporcionar educación para la salud, lo que es sumamente importante en el caso del enfermo, con factores de riesgo como los mencionados con antelación, pues se requiere de modificaciones en cuanto a estilo de vida y para que el enfermo tome consciencia de su enfermedad y le permita asumir autocuidado con relación a la nutrición, ejercicio, apego farmacológico, visita al médico, importancia del ejercicio, manejo del estrés. Además se verá reflejado en la disminución de costos sanitarios y en la reducción de reincidencias hospitalarias.

Es de suma importancia que el profesional de enfermería esté preparado para llevar a cabo, cuidados de calidad y hacer énfasis en la prevención de los factores de riesgo relacionados a la presentación de EVC a través de medidas de actuación multidisciplinar que incluyan un abordaje de los factores de riesgo cardiovascular modificables más significativos.

\section{BIBLIOGRAFÍA CONSULTADA}

1. Maldonado RNE. Atención al paciente con anticoagulantes. Rev Mex Cardiol 2001;9 (1-4): 44-9.

2. Blanch P, Ibernón M, Dos L, Padró J, Delso J. Identificación de factores responsables de anticoagulación oral excesiva en pacientes ambulatorios con cardiopatía. Rev Esp Card 2003;56(1):65-72.

3. Roca CAC, Muñoz LA, Castellar R, Garrido CR. Estudio del riesgo coronario y de ictus en pacientes hipertensos mayores de 54 años atendidos en atención primaria. Rev Clin Esp 2008;208(10):490-8.

4. Leiva PJ. Manual de urgencias cardiovasculares. 3ra ed. México: Mc Graw Hill/ Interamericana 2007.

5. INEGI/Secretaría de Salud. Dirección General de Información en Salud. Estadísticas de mortalidad general nacional. [serie en línea] 2005 [citado 19 de abril de 2010]. Disponible en: http:// www.sinais.salud.gob.mx/descargas/xls/m_005.xls

6. AHA. Soporte vital cardiovascular avanzado. Barcelona: Médica AWWE; 2006.

7. Jay HS. Medicina Interna. 3ra ed. México: Salvat;1991.

8. Barrón JV. Tratado de cardiología. 3ra ed. México: Intersistemas;2006.

9. Sanclemente AC, Pedragoza VA, Rovira PE, Vigil MD. El ictus en manos del internista. Factores de riesgo cardiovascular. Rev Clin Esp 2008;208(7):339-46.

10. Guadalajara JF. Cardiología. 6ta ed. México: Méndez Editores; 2006.

11. Bayés AL, Iwona C, Bayés GA, Grande C, Viñolas X, Rodríguez, et al. Espectro clínico y complicaciones de la fibrilación auricular. Rev Arch Card Méx 2004; 74, Suppl 2: 293-7.

12. Amariles P, Baena MI, Machuca M, Tudela J, Barris D, Sevilla J, et al. Conocimiento y riesgo cardiovascular en pacientes en tratamiento con medicamentos cardiovasculares. Ars Pharm 2005;46(3):279300.

13. Gómez GBP, Bautista SL. Detección de factores de riesgo cardiovascular y nivel de conocimiento de los mismos por el adulto. Rev Fac Med 2009; 52(6):248-52.

14. Córdova VJA, Lee GM, Hernández A MH, Aguilar SCA, Barriguete MJÁ, Kuri MP, et al. Plan de prevención clínica de enfermedades crónicas: sobrepeso, riesgo cardiovascular y diabetes mellitus 20072012 y sistema de indicadores de diabetes en México. Rev Mex Card 2009;20(1):42-5. 